\title{
A Realistic Approach to the Traffic Flow Behaviors by Cellular Automata Models
}

\author{
S.Rajeswaran \\ Department of Mathematics, \\ Research Scholar Sathyabama University, \\ Chennai, India
}

\author{
S.Rajasekaran \\ Department of Mathematics, \\ B.S.Abdur Rahman University, \\ Chennai-48
}

\begin{abstract}
Due to steady increase in traffic density on roads, the traffic phenomena become non-linear and complex. For the traffic engineers and policy makers to regularize the traffic flow, we need to explore the traffic behavior in detail. In the current paper, the usefulness of CA to traffic flow modeling has been determined. Also, some of the existing CA models have been extended to study characteristics of traffic flow that have not been captured either by using analytic models or by existing simulation techniques. We discuss CA models such as Standard Nagal model, Rule 184, Deterministic CA model, Stochastic CA model and Velocity Dependent Randomized CA model. We provide applications of Nagal model for urban streets and for freeway. Higher moments of traffic flow have been examined and their effect on overall traffic performance has been evaluated. We also examine the relationship between lane changing behavior and flow performance for a two-lane system. The ability of this modeling paradigm to capture the most important features of the traffic flow phenomena is established from the simulation results obtained.
\end{abstract}

\section{Keywords:}

Traffic flow modeling, Cellular Automata, one Lane Traffic ,Deterministic Model, Wolfram's Rule 184, Stochastic Model,VDR Model and A Two- lane Model.

\section{INTRODUCTION}

Of late, in metros, the traffic demand exceeded the capacity of vehicles. This results in increasing of pollution and increase of the number of accidents. One way to solve this problem is to construct additional roadways. Due to escalation of the constructing cost and environmental objection, this solution is impractical. Hence, man-made transportation systems have become more and more important for planning and prediction of traffic. The description of the characteristics and analytic technique of the fundamental traffic flow are provided by the theory of traffic flow. An important step in the design and control of transportation systems is to construct traffic flow modeling. Simplified models that capture the essentials of the dynamics of the transportation system play an important role in the research of traffic flow. A real possibility is to use CA models which are based on simple rules to simulate traffic in the microscopic level. These CA models are used to capture micro-level dynamics and relate these to macro-level traffic level
behavior.From a microscopic point of view, the process of a vehicle following its predecessor is typically expressed by a realtion which uses a stimulus- response [4]. In traffic CA models this response is the speed or acceleration of a vehicle.

\section{TRAFFIC MODEL CLASSIFICATIONS}

In the study of the impacts of different policies on vehicular traffic, modeling and simulations play on important role. In advanced countries modeling and simulations are used in intelligent information systems.

In general, there are two types of traffic models: Macroscopic and Microscopic.

Macroscopic models describe traffic with aggregate variables such as traffic density, mean speed, and volume. The use of such variables reduces the computation requirements for macroscopic modeling, making real-time calculation quite feasible. However, macroscopic models cannot estimate travel time, turning movements at intersections, fuel consumption, and control parameters on a short time scale.

Microscopic modeling considers the individual vehicle's physical status and the factors that control human driving behavior. The movement of individual vehicles is governed by the driver's behavior, the road topology, the status of surrounding vehicles, and the headway distribution. Each vehicle in the traffic may be described by a set of parameters that includes position, actual speed, desired speed, route choice, and willingness to pass the other vehicles.

\section{DEFINITION OF CA}

CA are mathematical models for dynamical systems in which space and time are discrete. CA consists of finite, regular grid of cells, each in one of the finite number of states. The grid can be of any number of finite dimensions. For each cell there is neighborhood that locally determines the evolution of the cell. The size of the neighborhood is the same for each cell in the lattice. The site value evolves synchronously in discrete time steps according to the value of their nearest neighborhood. These values are updated in a sequence of discrete time space according to finite fixed rule. The rule acts upon a cell and its direct neighborhood, such that the cell's state changes from one discrete time step to another.

\subsection{Mathematicians View}

Notation: $\mathrm{d}=$ dimension; $\mathrm{k}=$ states per site $\mathrm{r}=$ radius 
For simplicity, assume $\mathrm{d}=1$ for the moment.

A d-dimensional cellular automaton takes as its underlying space the lattice (integers). The dynamics are determined by a global function $F: S^{z} \rightarrow S^{z}$ whose dynamics are determined "locally" as defined below. A "local (or neighborhood) function" $\mathrm{f}$ is defined on a finite region as

$f: S^{2 r+1} \rightarrow S$, where $\mathrm{S}$ is a finite set of k elements.

The all-important property of CA is that this function is defined discretely. Both the domain and range of $\mathrm{f}$ are finite. The global function $\mathrm{F}$ arises from $\mathrm{f}$ by defining: $F(c)=f\left(c_{i-r}, \ldots \ldots \ldots, c_{i+r}\right)$

\section{IMPORTANCE OF CA MODELS}

1. CA models are conceptually simple, thus we can use a set of simple CA rules to produce complex behavior. Through the use of powerful computers, these models can encapsulate the complexity of the real world traffic behavior and produces clear physical patterns that are similar to those we see in everyday life. For example, CA models show the shifting from moving traffic to jamming traffic.

2. CA models can re-create all kinds of complicated traffic phenomena and reflect the properties of traffic flow.

3. Cellular automata models are capable of explicitly representing individual vehicle interactions and relating these interactions to macroscopic traffic flow metrics, such as throughput, travel time, and vehicle speed.

4. The massive parallelism and robustness of the model, which involves an efficient data processing and a high global tolerance in respect to local failures.

5. CA models can more adequately capture the complexity of real traffic, by allowing different vehicles to possess different driving behaviors (acceleration/deceleration, lane change rules, reaction times, etc.),

6. CA models, by being either deterministic or stochastic, can be more effective in accounting for the inherent variability in most real traffic.

7. CA models are amenable to representing both single and multilane traffic, which is particularly crucial for the modeling of highways.

\section{CA FOR ONE-LANE TRAFFIC FLOW}

In physical systems, space and time are discrete and physical quantities take on a finite set of discrete values. CA are realizations of physical systems. CA made up of a regular uniform finite lattice with discrete variables occupying various sites. At each site, the values of the variables completely determine the state of a CA. Based on a definite set of "local rule" and on the values of the variables in their neighborhood at the preceding time step the variables at each site are revised simultaneously [9].

We define the initial traffic model a 1-dimensional array with $\mathrm{L}$ cells and with closed boundary condition, which implies that $\mathrm{N}$, the total number of vehicles in the system, is kept as a constant. Each cell contains either one vehicle or none. Each cell corresponds to a road segment with a length $l$ equal to the average headway in a traffic jam. Traffic density $\rho$ is given by $\rho=N / L$. The velocity of each vehicle varies from 0 to $v_{\max }$. The number of sites a vehicle advances in one iteration is the velocity of each vehicle. The set of updating rules determines the movement of vehicles. At each iteration, to each vehicle these rules are applied in a parallel fashion.
As per the desired level of simulation detail the length of the iteration can be chosen. To approximate a continuous time system a sufficiently small iteration interval can be used. The distribution of vehicles among the cells and the speed of each vehicle in the cell determine the state of the system at each iteration.

The following notations are used to characterize each system state: $x(i)$ : position of the $i$ th vehicle,

$v(i)$ : speed of $i$ th vehicle, and

$g(i)$ : gap between the $i$ th and the $(i+1)$ th vehicle(i.e., vehicle immediately ahead) and is given by $g(i)=x(i+1)-x(i)-1$.

\section{DETERMINISTIC CA MODEL}

In the deterministic single lane model, vehicle motion is determined by the following set of updating rules:

Rule 1. Acceleration of free vehicles:

$v(i)<v_{\max }$ and $g(i) \geq v(i)+1 \Rightarrow v(i)=v(i)+1$.

Rule 2. Slowing down due to other vehicles:

$g(i)<v(i)+1 \Rightarrow v(i)=g(i)$.

Rule 3. Vehicle motion: Vehicle is advanced $v(i)$ sites.

These updating rules were first suggested by Nagel. Fig. 1 shows the application of these three updating rules to an example system with 24 cells and 7 vehicles:

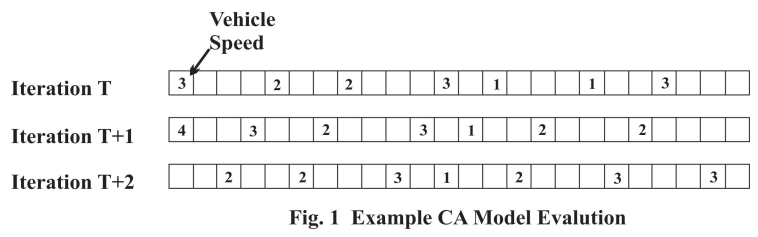

Note that in these rules it is assumed that all vehicles have same behaviors and have the same maximum speed. However, these assumptions can be relaxed. Let $v_{\max }=135$ cells/iteration and each iteration correspond to one second. Let the length of each cell be $7.5 \mathrm{~m}$. Since $7.5 \mathrm{~m} / \mathrm{s}$ is equal to $27 \mathrm{~km} / \mathrm{hr}$, vehicles assume the speeds $v_{0}=0 \mathrm{~km} / \mathrm{h}, v_{1}=27 \mathrm{~km} / \mathrm{h}, v_{2}=54 \mathrm{~km} / \mathrm{h}, \ldots$, and $v_{\max }=$ $135 \mathrm{~km} / \mathrm{h}$. The deterministic CA models are simplified versions of real traffic and these are useful modeling for automated highway systems where vehicle speeding and vehicle deceleration are externally controlled.

\subsection{Computer Simulation Of The Deterministic System}

The simulation is based on a system of 300 cells evaluated over 10,000 iterations (approximately 2.8 hours) for varying density levels. For each density level, various traffic flow measures were obtained (e.g., throughput, average speed, speed variance, traffic periodicity, etc.). The model reproduces the familiar fundamental diagram of flow versus density - see Fig.2. Flow is linearly increasing with initial increase in traffic density (laminar flow). A maximum flow of 3000 vehicles/hour is achieved at $\rho_{\max }=0.1667$, beyond which flow becomes linearly decreasing in density (back traveling start-stop waves). In the laminar flow phase, average speed converges to the maximum speed $v_{\max }$. 


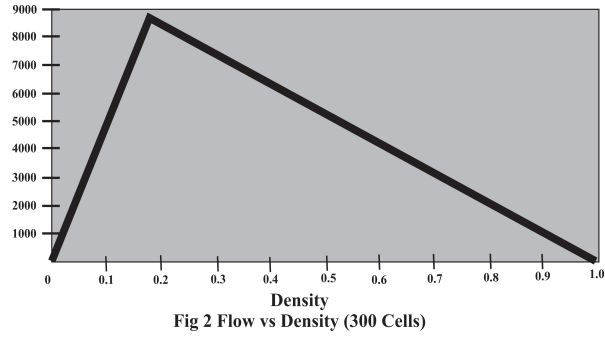

Average speed becomes a decreasing function of density for traffic densities larger than $\rho_{\text {max }}$ - see Fig.3.

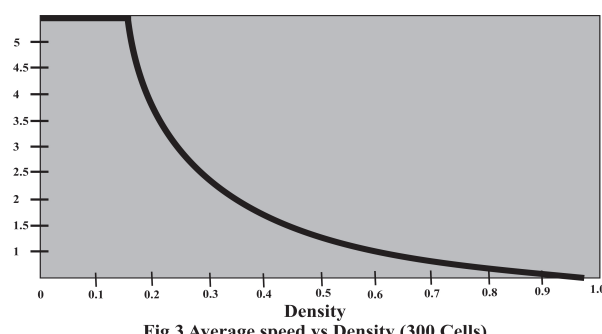

As shown in Fig.4 there is a maximum variance density which occurs shortly after the maximum throughput density. Thus, the region of maximum flow is also the region of maximum variance. Note that during the laminar flow phase, speed variance is negligible but starts to increase with the onset of maximum flow. The initial increase is fairly large creating a significant discontinuity in the variance-density function.

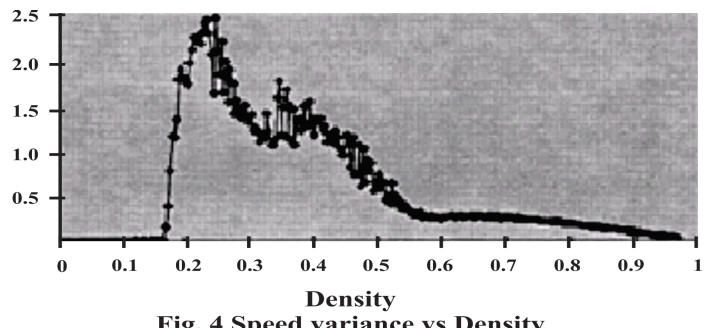

Fig.5 depicts the behavior of the period length as a function of density. Period length is negligible for densities below the maximum flow densities. Period length is negligible for densities below the maximum flow densities. However, the period length can be significant for higher densities.

In fact, either an increase or a decrease in period length may occur with an increase in density. More importantly, small changes in density could result in large fluctuations in period length.

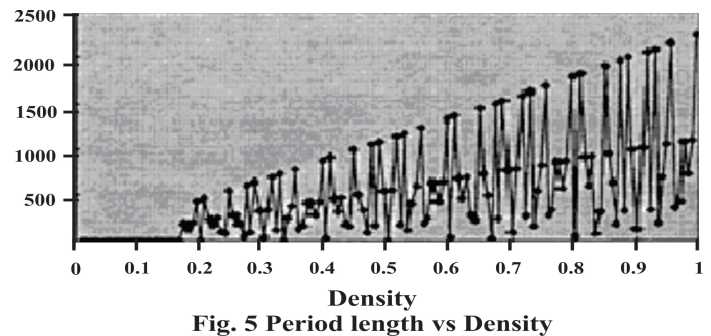

\section{THE STANDARD CA MODEL (STCA)}

\subsection{The Model and Its Rules}

In this model we use integer variables for space, time and speed. This kind of model is known as a cellular automaton[14]. The space is divided into cells so that the length of each cell is the maximum space headway between vehicles in jam and it is $1 / k_{j}$, where $k_{j}$ is the jam density so that if the length of each cell is $7.5 \mathrm{~m}$ then $k_{j}=$ $133 \mathrm{vel} / \mathrm{km}$. When the update time-step $\mathrm{t}=1 \mathrm{~s}$, the speed ranges from 0 to $v_{\max }=6$ cells $/ \mathrm{t}=162 \mathrm{~km} / \mathrm{hr}$. The STCA following the philosophy that goes as fast as you wish and as fast as the vehicle in front allows you and decelerate if you have to avoid a rear end collision $[7,8]$.

This philosophy is represented by the following four rules.

Rule 1. $v<v_{\max } \Rightarrow v=v+1$

Rule 2. $v>$ gap $\Rightarrow v=$ gap

Rule 3. $v>0 \Rightarrow v=v-1$ with $p_{\text {brake }}$

Rule 4. $x=x+v$

These rules can be updated in parallel for any vehicle. $p_{\text {brake }}$ is average deceleration ratio over the driver population. Average acceleration ratio over the driver population gives 1- $p_{\text {brake }}$. Though this model is extremely simple, it shows many features with the real-world traffic, STCA gives very good results, particularly in describing queueing system at the intersection of two urban streets $[2]$.

\subsection{Application of STCA Model For Urban Streets}

The capacity of the intersections is important for the urban traffic networks. By varying the parameter $p_{\text {brake }}$ this capacity can be reproduced very well using STCA.

Fig. 6 shows the shape of the capacity curves at intersections with traffic signals as function of the parameter $p_{\text {brake }}$. In the graph each curve is constructed by taking $p_{\text {brake }}$ along the $\mathrm{x}$-axis and saturation flow Qs (veh/hr) along the y-axis by keeping $v_{\max }$ at the intersection fixed (i.e., for various values of $v_{\max }$ we get various capacity curves). From the curve that corresponds to $v_{\max }=2 \mathrm{cell} / \mathrm{t}$ (i.e., $\mathrm{v}=54 \mathrm{~km} / \mathrm{hr}$ ), by taking $p_{\text {brake }}=0.2$, a saturation flow can be obtained. Hence, a parameter $p_{\text {brake }}=0.2$ should be applied in STCA to get a saturation flow of Qs $=1700 \mathrm{veh} / \mathrm{hr}$ for $v_{\max }=2$ cell/t. Also from these curves the move-up times $t_{f}$ (representing the average time headway between two vehicles departing at the stop line in succession) at intersections without traffic signals can be calculated. It is the reciprocal of the capacity at the stop line. i.e., $t_{f}=1 / Q s$.

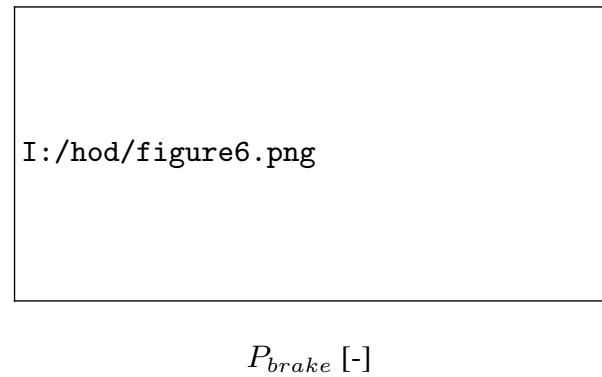

Fig. 6 Saturation flow Qs from STCA at signalized intersections 


\subsection{Application of STCA Model For Freeways}

On the other hand, as a result of the extreme simple configuration of the STCA, the characteristics of traffic flow in motion (no queuing) can be described by STCA with satisfying performance. The simulated traffic flow on motorways,cannot represent the realistic speed-flow relationships. Analyzing the STCA in details one finds the following unrealistic features on the microscopic level:

(i) The acceleration and deceleration ratios are unrealistic because of their infinite value and dependence on each other(acceleration ratio $=1-p_{\text {brake }}$, deceleration ratio $\left.=p_{\text {brake }}\right)$

(ii) The acceleration ratio $\left(=1-p_{\text {brake }}\right)$ is larger than the deceleration ratio $\left(=p_{\text {brake }}\right)$ (for the common case of $\left.p_{\text {brake }}>0.5\right)$.

(iii) The speed of a vehicle is not dependent on the speed of other vehicles ahead.

(iv) The minimum time headway between two vehicles cannot obtain values smaller than $t$.

(v) The driver reaction time is always equal to $t$.

(vi) The speeds are classified in discrete classes (i.e. with steps 27 $\mathrm{km} / \mathrm{h}$ ).

(vii) The threshold (time interval to the vehicle ahead) for changing (adjusting) speed is always equal to $t$.

These microscopic properties of the STCA lead to the following macroscopic disagreements compared to real traffic flow on motorways:

1. The maximum flow ratio in the opening (depressive) phase Copen is larger than the maximum flow ratio in the closing (compressive) phase Cclose, whereas in reality just the opposite occurs.

2. No capacity drops exist.

3. The convoy dynamics is always stable.

4. No breakdowns in an open pipeline system.

The first application of the CA for simulation of traffic flows on streets and highways was introduced by Nagal and Schreckenberg [7].

\section{WOLFRAM'S RULE-184}

Rule 184 has been popularised by Stephen Wolfram . This rule has been studied as the simplest 1-dimensional CA model for the highway traffic flow [12]. The rules are tabulated as below.

\begin{tabular}{|l|c|c|c|c|c|c|c|c|}
\hline current pattern & 111 & 110 & 101 & 100 & 011 & 010 & 001 & 000 \\
\hline new state for center cell & 1 & 0 & 1 & 1 & 1 & 0 & 0 & 0 \\
\hline
\end{tabular}

Here " 1 " indicates the cell is occupied by a vehicle and " 0 " indicates that the cell is empty. According to Rule 184, the movement of a vehicle in a cell depends on its two immediate neighborhoods. If the vehicle has an empty space in front of it, it will move one unit to the right. Otherwise, it remains in the original cell. Under this rule, the number of filled cells does not change.

The following time-space diagram (Fig.7) of CA-184 shows that it constitutes a fully deterministic system that strictly repeats itself.

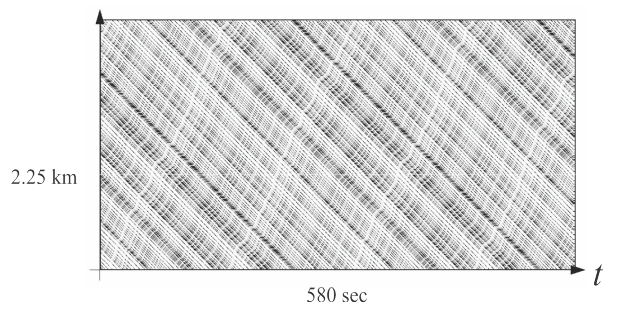

Fig. 7 A typical time-space diagram of the CA-184 TCA model. The global density of the system was set to 0.3 . There are many deterministic congestion waves present; they have an eternal lifetime in the system.

\section{STOCHASTIC CA MODEL (SCA)}

Since this model needs some fluctuations for explaining traffic jams, the presence of a noise parameter, represented by the slowdown probability, is required. From the following time space diagram(Fig.8) of the SCA model one can see that the breaking behavior creates mini jams which are not so in real-life observations.

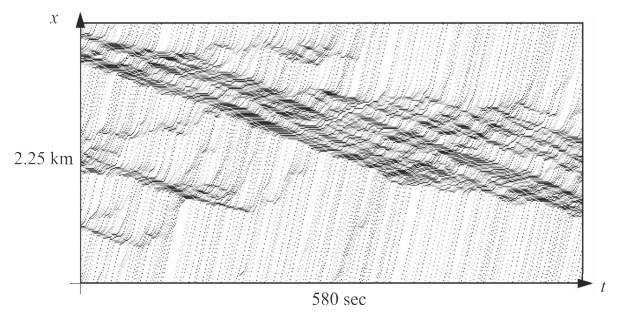

Fig. 8 A typical time-space diagram of Nasch's SCA model. The slowdown probability was set to 0.5 and the global density of the system to 0.2 ; the large congestion wave is very raveled out, resulting in many mini-jams localized in its neighborhood.

\section{VELOCITY DEPENDENT RANDOMIZATION MODEL (VDR)}

By reducing the outflow, one can achieve the stable traffic jams. The reduction in outflow is made possible by implementing the slow-tostart rule, by which we mean that vehicles in the front portion of the jams are made to wait a small amount of time. One such example is VDR Traffic CA [1].

This model is based on the following rules:

The rule steps for the vehicle $\mathrm{n}$ of this model are:

1. $v_{n}(t+1):=\min \left(v_{\max }, v_{n}(t)+1\right)$

2. $v_{n}(t+1)=\min \left(\frac{d_{n}(t)}{\Delta t}, v_{n}(t)+1\right)$

3. $v_{n}(t+1):=\max \left(0, v_{n}(t+1)-1\right)$, with probability $p(v)$

4. $x_{n}(t+1):=x_{n}(t)+v_{n}(t+1)+\Delta t$

where $p=p(v(t))$, a function of vehicle speed and $d_{n}(t)$ represents the number of empty cells in front of the $n t h$ vehicle. The braking probability is calculated from to the gap (the number of empty sites) in front of the vehicle $n$ and the braking probability is calculated from

$$
p(v)=\left\{\begin{array}{l}
p_{n}, \text { for } v=0 \\
p, \text { for } v>0
\end{array}\right.
$$

This gives the characteristics of flow almost coincide with realistic value. The system settles into a phase several state with a large jam and a free flow zone. This can be seen from the following time space diagram(Fig.9). 


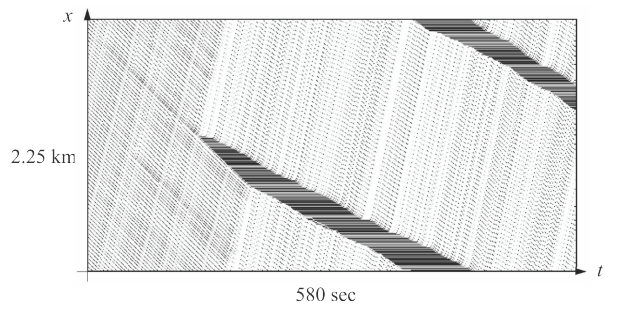

Fig. 9 A typical time-space diagram of the VDR CA model. The slowdown probability was set to 0.01 , the slow-to -start probability to 0.5 and the global density to 0.2 . We can see the formation of a persistent compact jam; there's also a significant decrease of the density in front and behind the jam'sformation.

\section{A TWO-LANE CA MODEL}

All the previous work dealt with unidirectional roads. Some authors, e.g., Gundaliya et al. [3], Mallikarjuna and Ramachandra Rao [5] used multi- cell structures. In real life situation vehicles moving in multi-lane road. Hence there is a need to have a model for two-lane traffic. A two-lane model due to Nagal and schreckenerg consists of two parallel single lane models with periodic boundary conditions. The following rules have to be observed before changing the lane: .

Rule 1: Check ahead your current lane if another car is in your way.

Rule 2: Check ahead on the other lane if it is better there

Rule 3: Check back on the other lane if you would get in the way of another vehicle.

Rule 4: Based on the result of the first three rules, decide whether to remain on the same lane or change to the other lane.

Using these rules and the algorithm for the one-lane model the following conditions have been arrived for a vehicle to change a lane:

Rule 1. gap $_{i}<v_{i}+1$

Rule 2. gap $_{i}^{\prime}>v_{i}$

Rule 3. gap $_{i}^{\prime \prime}>v_{\max }$

To bring stochasticity into the algorithm we add the additional condition "rand ()$<P_{\text {noise }}$ is the probability of lane changing. Before the velocity update this step on lane changing to be implemented.

\section{DISCUSSION AND CONCLUSION}

The usefulness of CA to traffic flow modeling has been explored. Existing CA models have been used to capture interesting characteristics of traffic flow that has not been possible using conventional analytic models or existing simulation techniques.Traffic CA models provide us with sufficient detail in order to capture the microscopic nature of traffic flows. Higher moments of traffic flow were examined using CA models.For example, we showed that the density area of maximum throughput is also the density area of maximum speed variance. For deterministic systems, we found traffic flow to possess a finite period which is highly sensitive to density in non-monotonic fashion. In this paper, it has been shown that CA models are more suitable to modeling multi-lane traffic. In this model, lane changing rules and behavior are explicitly accounted. The impact of these rules and behaviors becomes then easier to examine. We observed that lane changing does little to increase throughput. Since more frequent lane changing means an increase in the likelihood of traffic accidents, traffic should be smaller than both the density of maximum lane changing and maximum throughput. This will ensure traffic with few lane changes and with a small speed variance. Simulation results indicate that (i) the essential feature of traffic flow can be captured by this method and (ii) this model is useful for application to large scale traffic networks.

\section{REFERENCES}

[1] Barlovic ,R., Santen,L., Schedschneider,A. and Scheckenberg, M.(1998): Metastabe states in Cellular Automata for Traffic Flow, European Physics Journal, Vol B5, nr 793.

[2] Brilon,W.and Wu,N.(1997): Calibration and Validation Cellular Automaton. Technical Report,PlaceTypeInstitute of PlaceNameTraffic Engineering,Ruhur-University CityplaceBochum.

[3] Gundaliya,P., Tom,V., and Dhingra,S.(2004) Heterogeneous traffic flow modeling using cellular automata for an arterial, in: Proceedings of the 6th International Workshop on Transportation Planning and Implementation Methodologies for Developing Countries (TPMDC04), Transportation Systems Engineering, IIT Bombay, 2004.

[4] Maerivoet,S. and De Moor, B. (2005), Transportation Planning and Traffic Flow Models, 05-155, Katholieke Universiteit Leuven, Department of Electrical Engineering ESATSCD (SISTA).

[5] Mallikarjuna,Ch., Ramachandra Rao,K. (2005): Traffic Flow Modeling on Highways using CellularAutomata, A Review, Proceedings of International Conference on Structural and Transportation Engineering, (START-2005) (Eds. J.N. Bandyopadhyay and B. Maitra), Elite Publishing House, placeN. Delhi,pp 912-919.

[6] Nagel, K. and H. Hermann,(1993) "Deterministic Models for Traffic Jams: Physica A, Vol.199, pp. 254-263.

[7] Nagel, K. and M. Schreckenberg,( 1992) "A cellular Automaton Model for Freeway Traffic," Journal de Physique,Vol 2, pp.2221-2229.

[8] Nagel, K.(1995) : High-speed microsimulations of traffic flow, Ph.D theis, University of Cologne.

[9] Nagel, K.( 1996): Particle Hopping Models and Traffic Flow Theory, Physical Review E,Vol. 3, No. 6, pp. 4655-4672.

[10] Schadschneider, A. and M. Schreckenberg(1993): Cellular Automaton Models and Traffic Flow, Journal of Physics A, Vol. 26, pp. 679-683.

[11] Schreckenberg, M., schreckenberg, A., Nagel, K. and N.Ito(1995): Discrete StochasticModels for Traffic Flow, Physical Review E, Vol. 51, No. 4, pp 2939-2949.

[12] Stephen wolfram (2002) : A New kind of Science, Wolfram Media, Inc., ISBN 1-579-955008-8.

[13] Villar, L. and A. de Souza(1994), "Cellular Automata Models for General Traffic Conditions on a Line," PhysicaA, Vol. 211, pp. 84-92.

[14] Wolfram,S.(1986): Theory and Applications of Cellular Automata.World Scientific country-regionplace Singapore. 